\title{
Sir Ludwig Guttmann Lecture
}

\section{Neuropathology: the foundation for new treatments in spinal cord injury}

\author{
BA Kakulas*,1,2 \\ ${ }^{1}$ The Australian Neuromuscular Research Institute, Queen Elizabeth II Medical Centre, Nedlands, Western Australia; \\ ${ }^{2}$ Centre for Neuromuscular and Neurological Diseases, University of Western Australia, Western Australia
}

The first step essential in the search for a cure of human spinal cord injury (SCI) is to appreciate the complexity of the disorder. In this regard, it is not only the loss of ambulation but the sensory and autonomic changes that are equally important in recovery. In addition, there are the serious social emotional psychological and lifestyle effects of SCI which should also be taken into account. It is also true that no two SCI lesions are alike as each is the result of a SCI unique to that individual. Clinically of utmost importance is the segmental level of injury and whether it is complete, incomplete or discomplete (loss of all neurological functions below the injury but with physiological or anatomical continuity of Central nervous system tracts across the lesion). We are not concerned here with primary and secondary prevention or methods designed to limit the severity of the lesion after the event, important as they are, but with the requirements for a cure. Clearly, the greater the number of nerve fibers that can be preserved in the acute stage, the better will be the end result. Our focus at present is on the end-stage lesion with the aim of showing that a cure for SCI will depend upon establishing functionally useful central axonal regeneration and reestablishing physiological reconnections. Existing experimental methods are based on stimulating axonal regeneration by neutralizing inhibitory factors, adding positive trophisms and creating a permissive environment. Better results are obtained by bridging the gap with grafts of peripheral nerves or transplants of Schwann cells and genetically engineered fibroblasts. Recently, the potential for stem cells to enhance this process has created great interest. This is because of the ability of pluripotential cells to differentiate into neural tissue. A cure based on the physiopathology of SCI requires pyramidal, extrapyramidal, sensory, cerebellar and autonomic pathways to be regenerated with their appropriate neurotransmitters restored and reflexes integrated physiologically and in synchrony. In human SCI, there is a very long distance anatomically for axonal regrowth to occur in order to reach their relevant nuclei. This is because of continuing Wallerian degeneration. It also presumes that the target neurons are intact and that there has been no transneuronal degeneration above or below the lesion. Alternatively, in place of regenerated long axons, a multisynaptic pathway may be constructed from stem cells that have developed into neurons. Whether such a pathway would restore useful neurological functions is unknown. At present, the transplant and grafting research teams are exploring these possibilities in experimental animals. Moderate success in gaining axonal regeneration has been reported; however, it must be appreciated that the human lesion differs considerably from that of the experimental animal. In order to be successful, the neuropathology and neurophysiology of human SCI must be taken into account. The purpose of this review is to place the requirements for a cure, using stem cells, within the context of the neuropathology of human SCI.

Spinal Cord (2004) 42, 549-563. doi:10.1038/sj.sc.3101670; Published online 31 August 2004

Keywords: spinal cord injuries; stem cell therapy; neuropathology

*Correspondence: BA Kakulas, Australian Neuromuscular Research Institute, Queen Elizabeth II Medical Centre, Nedlands, 6009, Western Australia

The Sir Ludwig Guttmann Lecture, 43rd International Spinal Cord Society (ISCoS) Annual Scientific Meeting, Athens, Greece, 26-29 September 2004

\section{Introduction}

It is timely to consider whether a cure for human spinal cord injury (SCI) will be found within the foreseeable future in the light of the many recent advances in experimental neurobiology especially that which relates to stem cells. It is now well known that central axonal regeneration is possible under certain experimental 
conditions in mammals. Inhibitory molecules are inactivated and a 'permissive environment' created enhanced by the addition of trophic factors, peripheral nerve grafts and cellular bridges. As stem cells are able to differentiate into central nervous system (CNS) tissues, the experimental possibilities are even wider. A cure for SCI based on stem cell technology is an exciting possibility and there is general optimism that this will soon be found.

The fact that a cure may even be considered is a compliment to the workers concerned and a credit to the organizations that support their research. It was not so long ago that a spinal injury was a virtual death sentence little different to the historical reference that it was 'a condition not to be treated'. ${ }^{1,2}$ This terrible prognosis no longer applies as a result of the magnificent work of Sir Ludwig Guttmann in the UK followed by Sir George Bedbrook in Australia, both of whom revolutionized the treatment of SCI. These men are recognized internationally as the pioneers of the new era and we pay special tribute to Sir Ludwig in whose memory this Lecture is named and which I am honored to give. It is now timely to consider whether we can go further than good clinical management and bring about a cure based on stem cell research.

Highlighted in this review is the complexity of the neuropathology of SCI. Although there is great urgency for the results of recent research to be applied to the patient, it is strongly recommended that human trials should not begin until the experimental methodology is thoroughly worked out in a suitable animal model. There is a real possibility that by rushing into human trials the quest will be hampered in the long term because of poor planning. It is a cliché that most people think of a cure for SCI as a return of simple ambulation not recognizing that the loss of sensation may be equally disabling as paralysis. Autonomic disturbances are also very important and contribute to the overall disability. For instance, there is a vast difference between paraplegia due to poliomyelitis which spares the sensory and antonomic systems and SCI where there is loss of all neurological functions.

Given this brief and sober introduction, the relevant neuropathological features of human SCI will now be considered in the light of the requirements for a cure using stem cell technology in conjunction with other recent advances in research.

\section{The neuropathology of human SCI}

\section{General}

The first point to bear in mind is that each SCI patient is different so that any treatment regime will need to be customized to that particular patient. This individuality applies both to the injury of the vertebral column and to the spinal cord itself. For instance, in some cases of vertebral injury the spinal cord escapes altogether. At other times, the spinal cord may appear normal to the naked eye but will be extensively damaged microscopi- cally in cases in which the vertebral column is relatively intact. Usually in SCI, the spinal cord is found to be contused, compressed, lacerated, disrupted and (rarely) transected. The SCI may be minor, moderate or severe, and involve multiple levels extending over several segments. Typically, in flexion injuries the central gray matter undergoes hemorrhagic necrosis a reflection of its rich blood supply. Edema and petechial hemorrhages are found in the white matter tracts. The vertebral column injury is associated with a greater or lesser degree of hemorrhage within the extradural, subdural or subarachnoid spaces and the covering membranes are torn. In our autopsy protocol, the bony column and spinal cord are removed together, so that it is possible to reconstruct the injury and relate the bony to the soft tissue changes., ${ }^{3,4}$

The mechanism responsible for the SCI is direct physical compression, with the amount of rotation distraction and distortion varying according to the type of the injury. Vertebral injuries are classified as flexion, extension, rotation, distraction and compressive in type. In each case, the spinal canal is compromized thus injuring the cord. However, it is unusual to find detached fragments of bone which could aggravate the cord lesion. Although hemorrhages within the various meningeal compartments are common, hematomas rarely cause significant compression of the cord. A feature of the vertebral injury is that realignment of the vertebral column often occurs spontaneously following the event and the capacity of the spinal canal restored.

The microscopic neuropathology of the spinal cord following injury is well known. ${ }^{5}$ The natural history is more or less stereotyped according to the usual cellular events that occur in the CNS after trauma. The morphology varies according to the stage examined being acute, subacute or chronic. Obviously any 'curative' treatments should be specifically designed for the stage for which the intervention is planned (see below).

Clinically, injuries are said to be (neurologically) complete, incomplete or discomplete depending on the presence or absence of neurological functions below the level of injury. At the segmental level, the paralysis is of the lower motor neuron (LMN) type resulting from the central gray matter destruction and/or associated nerve root damage or peripheral nerve trauma. However, it is the disruption of the long white matter tracts that are much more important in determining the clinical picture in SCI. Below the lesion, paralysis is of the upper motor neuron (UMN) type with increased muscle tone (spasticity), exaggerated reflexes and positive Babinski sign. An exception occurs in lesions of the cauda equina in which case paralysis is of the flaccid LMN type. This is because the roots of the cauda equina are capable of regeneration being peripheral in type, a very different situation compared with the cord proper. In general, the rehabilitative outcome of SCI is determined by the severity of the neurological disorder. The neurological picture in life can be precisely correlated with the 
pathology resulting from the injury because of the segmental nature of spinal cord anatomy.

It is of interest that despite the massive forces involved in causing the vertebral injury and not withstanding its very delicate consistency, the spinal cord is relatively resistant to the initial trauma. However, the cord is very vulnerable to secondary compression because of the instability of the column consequent to the injury. This calls for very careful handling of suspected SCI patients. Aggravation of injury is all too common even when the patient is in hospital.

The following data are illustrative of these points. In a series of 588 coronial autopsies of spinal injury, 162 $(27.6 \%)$ had detectable injury of the vertebral column but showed no evidence of damage of the spinal cord. In survivors such people are said to have had 'a lucky escape'. In the same series there were eight cases $(1.36 \%)$ in whom the vertebral column including discs and ligaments were apparently intact but the spinal cord was visibly injured. In the majority of cases of the series there was damage both to the spinal cord and vertebral column. Noteworthy was the observation that transection of the cord was uncommon being found in 84 of the 588 cases $(14.3 \%)$. The remainder had some continuity of spinal cord tissue across the lesion.

Within this coronial series of 588 individuals, there were 219 persons who survived for at least $24 \mathrm{~h}$. It was possible to assess the clinical neurological status of these 219 patients. Of these, $44(20.1 \%)$ were incomplete, 39 $(17.8 \%)$ complete and $53(24.2 \%)$ discomplete, that is, showing some anatomical continuity of spinal cord parenchyma across the lesion but who were clinically neurologically complete during life. The clinical status was unknown in $83(37.9 \%)$ of these short-term survivors. In no instance in this series of 426 cases with spinal cord damage was there sufficient extradural, subdural or subarachnoid hemorrhage present to cause significant compression of the spinal cord.

Another notable feature of the human material is that traumatic demyelination is uncommon being found in only 18 cases. However, this is not a definitive figure since in order to find bare axis cylinders many frozen sections require to be examined microscopically and at different levels. But it may be taken that traumatic demyelination is not immediately obvious in human material compared to animal models. Indirect evidence of traumatic demyelination can be deduced from Schwann cell remyelination as was found in 38 cases in the series. These patients had central axons remyelinated with Schwann cells, which is presumptive evidence of previous demyelination.

In the acute phase, permanent or transient axonal damage is demonstrated histologically by immunochemical display of accumulated 'A Beta' protein, a component of the amyloid precursor protein or APP within the axons. These axons have had their transport system interrupted, but a proportion are capable of recovery. Those which do survive may thus have been remyelinated with peripheral Schwann cell type myelin. ${ }^{6}$ Other bare axons are found to be disrupted with terminal bulb formation. Early on these axons may show 'abortive attempts at regeneration' with growth cones in evidence, but they soon degenerate. ${ }^{6}$

\section{Natural history of human SCI}

The first sign of injury is edema of the cord, manifest as swelling, due to exudation of fluid from capillaries. This occurs within minutes following injury and is usually accompanied by parenchymal hemorrhages of the white matter. Necrosis of the central gray matter is the usual outcome in flexion and extension injuries. 'Solid cord injury' without visible hemorrhage or overt disruption of parenchyma is uncommon. Clinically, this type of injury is associated with a 'central cord syndrome', the principal lesions being in the white matter of the lateral columns adjacent to the central gray matter. ${ }^{7}$

In this acute phase, necrosis and to a lesser degree apoptosis is responsible for death of neurons and glia in the vicinity of the injury. There is also the effect of excitotoxic shock with havoc created by free radicals at the molecular level. Reactive vascular changes initiated by cytokines released from the damaged tissues may be in evidence in the first $12 \mathrm{~h}$ represented by emigration of polymorphonuclear leucocytes and vascular dilatation. Lymphocytes appear soon afterward and macrophages are also present within 24 to $48 \mathrm{~h}$ of injury. The number and proportion of these cells varies greatly from case to case. Within $72 \mathrm{~h}$ fat-laden macrophages are abundant. These cells ingest myelin fragments converting them enzymatically to neutral fat for removal from the site. Reactive changes are believed to be responsible for secondary damage to the spinal cord parenchyma, which may at first have appeared apparently intact.

This describes the natural history of acute phase of the posttraumatic lesion in SCI. The next, subacute stage is characterized by cavity formation resulting from removal of debris by the continuing macrophage activity. Astrocytes that first appear about 5 days after injury proliferate and lay down glial fibers forming an irregular 'heteromorphic' network. Depending on the extent of arachnoid and dural tearing, collagenous fibrosis may be prominent within and surrounding the cord lesion so that the subarachnoid space is obliterated. The amount of collagenous scar formation is variable and tends to be in proportion to the amount of hemorrhage.

Beyond the level of immediate injury, there are many changes taking place in the remaining cord tissues. Wallerian degeneration of axons is a continuing process distal to the neuronal cell body. As time passes, the axons distal to the injury disintegrate and their myelin sheath breaks up into globules. Axonal degeneration and myelin breakdown is found caudal to the lesion in the motor pathways and rostral in the sensory tracts. Macrophages take up the debris and astrocytes lay down fibers in parallel to the lost axons resulting in 'isomorphic' gliosis. Wallerian degeneration is well established in patients who survive 12 months or more. Other distant changes are more subtle consisting of 
reflex aberrations and the 'plastic' neurophysiological effects at the synaptic level arising from changes in the excitory or inhibitory signals, thresholds, neurotransmitter release and neuroendocrines. These plastic changes are continuous.

In the chronic or end stage of SCI, the lesion typically consists of a multilocular cavity traversed by vascularglial bundles accompanied by a regenerated nerve roots. Astrocytic and collagenous fibers in various degree of admixture run across and surround the cavities. The multilocular cystic cavities are not related to posttraumatic syringomyelia but merely represent the removal of debris by body's healing processes. The proportional amount of gliosis, collagen, new blood vessels, macrophages (some iron laden) regenerated nerve roots and the remainder of the original spinal cord (CNS) tissue differs in each individual SCI.

The possibility that a limited amount of central axons regeneration has occurred spontaneously cannot be excluded, but impossible to demonstrate directly in human post-mortem material. Central axons myelinated by Schwann cell can be found but these may either be remyelinated as described above or possibly central axons induced to regenerate by the Schwann cells. The lesion in this end stage is more or less static except for the continuously advancing Wallerian degeneration of spinal tracts above and below the lesion and the effects of plasticity on the neuronal synaptic network. Nerve roots also continue to grow in a random fashion both within the remaining parenchyma and outside the cord. The other consequence in the long term is posttraumatic syringomyelia, which develops in about $5 \%$ of cases. Other late complications are due to the aging process such as atheroma of feeding arteries and intervertebral disc degeneration. This change and ligamentum flavum hypertrophy may cause spinal stenosis. These effects may be responsible for a changing neurological picture in the long term. ${ }^{3}$

\section{Clinicopathological correlation}

The neurological status of each patient reflects the neuropathology of the lesion with which an exact neurological correlation may be made because of the precise segmental anatomy of the spinal cord. Absent or partially retained neurological functions are determined by the segmental level and by any of the residual white matter tracts that have survived the injury. However, there is one proviso to this guideline in that there is a point where such remaining axonal fibers, although in continuity across the lesion, are too few to allow for retained voluntary motor activity below or above for sensation. Signals transmitted across the lesion in otherwise complete injuries giving rise to the term 'discomplete' and correlate well with the morbid anatomical finding of preserved white matter bridging the lesion. ${ }^{6}$ These discomplete patients provide a fertile ground for the restorative neurologist, who is concerned with exploiting and enhancing residual functions. ${ }^{8}$
The neurological status of any one SCI patient is not settled until about 3 weeks or more following the injury and some recovery can be expected up to a year. The basis for early recovery is resolution of edema and return of function in axons that were only partially injured. In addition to the overt neurological deficits, the clinical picture in each case is complicated by unwanted abnormal neurological activity. These consist of socalled 'spasms' (reflex contractions) and covert painful burning sensations, distortion of (phantom) body image and other disconcerting effects. These unwanted side effects of SCI are believed to be caused by enhanced excitory or subdued inhibitory neuronal thresholds combined with the effects of plasticity of the CNS tissues. New connections are created in response to afferent inputs arising from sensory nerve endings in the skin and sensory organs of the joints, ligaments and muscles. Reflex contractions are also aggravated by urinary tract infections, pressure sores and the like which increase the sensory input. Adding to these mechanisms are the aberrant neuronal connections resulting from the injury. The postulated microanatomical and known neurophysiological changes that take place in the neuronal network in the segments below the lesion following SCI are dynamic, complex and continuously evolving.

These correlations apply principally to MVA, diving and sporting accidents and only partially to gunshot or knife wounds in which the injury is more defined and complete so that principle of initial relative resistance of the spinal cord to the injury no longer holds. In knife wounds and those caused by sharpened bicycle spokes, prominent in Africa, differ from MVA trauma since there is a 'clean' transection. However, the neuropathological sequence is similar with reactive cellular changes at the level of the lesion, Wallerian degeneration distally and altered neurophysiological functions above and below.

\section{Special features of the neuropathology of SCI}

Quantification An investigation of the minimum number of preserved axonal fibers required to retain some voluntary motor function, no matter how little and the minimum number required for conscious sensation was evaluated in selected post-mortem cases. ${ }^{2,5}$ In one 'incomplete' patient with retained motor function of the ankle there was only $1.17 \mathrm{~mm}^{2}$ of retained white matter at the level of the lesion, whereas in complete SCI patients there may be as much as $3.89 \mathrm{~mm}^{2}$ spared. Clearly, there is no direct correlation with function, which is not unexpected as the site and quality of preserved white matter would be quite variable. Similarly, an attempt was made to quantify the minimum number of fibers associated with retained motor and sensory functions. In six clinically incomplete cervical injuries, the descending axonal fibers in the corticospinal tracts of lateral columns were counted at T4. The lowest number of axons found in an incomplete patient with minimal motor function below the level of 
the lesion was 3175 (normal control 41472 ). This patient had retained plantar flexion of the right foot. The lowest number of fibers counted in the posterior columns in a sensory incomplete patient was 117359 (normal control 452480 ). Thus by quantifying the number of axons that traversed the lesion, it was possible to estimate the minimum number of axons needed to restore function in regeneration experiments. ${ }^{2,9}$ Although approximate due to the limitations of the methodology, these results provide a useful indication of the number of axons required to restore voluntary motor activity and conscious sensation within the framework of planning a curative treatment.

Similarly, a study was undertaken to investigate the type of myelination, central or peripheral of remnant axons within the lesion. Of great interest in this study was the finding of many central axons myelinated by Schwann cells. These were interpreted as either being surviving axons that had lost their myelin sheath, that is, traumatic demyelination and remyelinated with Schwann cells, or regenerated central axons. Theoretically, under the trophic influence of the Schwann cells these axons may indeed have regenerated. As this work was undertaken on human post-mortem material, it is not possible to test this possibility experimentally as it would require in vivo neuronal transport markers to be used. 5,6 The limited amount of clinical-relevant correlation with amount of remyelination is as follows.

Observations of human central axonal remyelination in human SCI were taken from a study of 27 cases selected from the Royal Perth Hospital collection. These patients survived at least 6 months after sustaining their spinal injury. Tissues were taken from the area of disrupted cord and sections stained with luxol fast blue/ periodic acid Schiff (LFB/PAS), which distinguishes by color peripheral (deep blue) from central (green) myelin. Of the 27 cases, 16 showed central axonal remyelination by peripheral Schwann myelin in the LFB/PAS-stained sections. The age range of these 16 patients showing remyelination was from 12 to 52 years. Post injury survival ranged from 1 to 38 years. In all, 13 were cervical injuries (C5-C7) and three thoracic (T4, T6, T12/L1). All tissue sections were at the level of the lesion. A cystic cavity due to extensive loss of normal cord parenchyma with gliotic walls containing these residual axons and their myelin sheaths among vascular bundles form the common lesion. This lesion was present in 14 out of the 16 cases showing remyelination and nine of 11 of the non-remyelinated cases. The remyelinated central axons are morphologically different to peripheral nerve root regenerations and readily distinguishable from them. Central remyelinated axons occur in clusters or as single remyelinated axons generally in the center of the lesion in close proximity to the cavity, but occasionally they are found near dorsal roots and in the subpial region. In longitudinal sections, it is possible to find many axis cylinders surrounded by peripheral-type myelin several millimeters above or below the lesion as well as at the site of injury. Some cases show peripherally myelinated axons encircling a new blood vessel within the reactive changes. Remyelinated axons are often tortuous following the deformity of the cord parenchyma at the level of the lesion. All cases with remyelination also had identifiable nerve root regenerations. ${ }^{6}$

\section{Regenerated nerve roots and late recovery}

In most SCI patients who survive for more than a year, regenerated nerve roots singly or in bundles can be found. Thus, there were 37 cases with nerve root regeneration in a series of 75 survivors of more than 6 months in one of our early studies. Age, sex and level did not influence this incidence. The nerve roots are usually present in heteromorphic bundles akin to a traumatic neuroma and arise from both posterior and more commonly anterior roots. Despite their proliferation a clinically detrimental 'space-taking' effect was not observed in any of these patients. These nerve roots grow continuously and are Schwann cell myelinated. However, there is no evidence that they exert any trophic influence on central axonal regeneration except for the tantalizing question of the mechanism responsible for late recovery in some rare instances of SCI. This phenomenon has many alternate explanations, the best being plastic reorganization of residual CNS elements under the influence of intense sensory stimulation usually provided by skin massage. 5,6

This describes in essential detail the neuropathology of human SCI. Those who wish to read further are referred to Hughes (1978) ${ }^{1}$ and Wolman (1965), ${ }^{10}$ who have produced excellent monographs on the neuropathology of human SCI in earlier times. More information can be derived from Tator ${ }^{11,12}$ who provides a more recent account of the neuropathology of SCI and also draws attention to secondary or continuing damage after the event of injury. He also deals with the neuropathology of spontaneous recovery in human SCI. It is well known clinically that most neurological recovery occurs within the first 3 weeks of injury and that some recovery can be expected in up to a year. It has also been reported that neurophysiological studies in the early stages may predict the final neurological outcome following SCI in incomplete cases. ${ }^{13}$

\section{The requirements for a cure in the light of the neuropathology of SCI}

The voluntary motor system

In principle, the aim is to reconnect the long CNS pathways both efferent and afferent, which were disrupted by the injury. The corticospinal pathway (pyramidal tracts) originates in the motor cortex and consists of the very long Betz cell axons. Our qualitative study of Betz cells has shown that they are retained in long standing SCI. In experimental studies, it has been found that the neuronal body will degenerate if the axonal disruption is close to the soma. Fortunately they are distant in SCI. Corticospinal axons are subject to 
limited retrograde atrophy proximal to the lesion for an unknown extent but not likely to be more than a few millimeters, as judged from myelin-stained sections from above the lesion. Axonal signals arising in the motor cortex and transmitted by the pyramidal tracts are modulated by inputs from the basal ganglia and cerebellum and integrated with the extrapyramidal systems to form the final 'common path' leading to the anterior horn of the spinal cord. The corticospinal anterior horn motor neuron connection is monosynaptic. At the segmental level, the motor neurons of the gray matter are further influenced by signals from the muscle spindles. This reflex is responsible for reciprocal innervation of agonist and antagonist muscles. Below the lesion in SCI the corticospinal axons are lost over long distances due to Wallerian degeneration, which is a continuous process. ${ }^{14}$ The gradual loss of myelin due to Wallerian degeneration is accompanied by astrocytic scar formation. ${ }^{15}$ The problem of recreating these pathways is therefore manyfold and presents a formidable task to the experimental neurobiologist concerned with axonal repair.

Theoretically, the first approach in gaining a cure is to reconnect the long pyramidal axons with motor neurons in the relevant segments below the lesion hoping that such central axons will grow through the scar and reestablish synapses. Alternatively, a multisynaptic pathway may be created running from the ends of the corticospinal axons to the anterior horn cells below. Because of the many synapses this new pathway would be slow conducting. What effect this might have on restored function is unknown, but one would hope that some useful motor functions would be restored in this way. In either case, a serious consideration is to ponder whether such restored axons would ever reach their correct segmental level and make appropriate connections with the motor neurons and thus restoring voluntary motor control. It is helpful in this respect that a 'central pattern generator' for locomotion is known to exist in the human lumbar spinal cord. This pattern generator combined with the known detailed neurophysiology of normal ambulation forms a useful guide to those concerned with restoring motor function. ${ }^{16,17}$ It also gives hope to the view that a useful outcome being derived from a minimal UMN input.

\section{The extrapyramidal system}

Similar considerations apply to the long efferent extrapyramidal descending pathways such as the reticulospinal, tectospinal and vestibulospinal tracts. These involuntary motor tracts control posture and equilibrium, and are equally important as the voluntary system in the production of normal movement. As such they will also require repair if a cure is to be achieved.

The sensory system

Again, it is a matter of regenerating the long axons of the posterior columns for touch vibration and position sense, ensuring that the new fibers reach their appropriate destinations in the brain stem and thalamus. In common with the pyramidal tract, a certain amount of retrograde axonal degeneration is expected to occur below the level of the lesion and considerably more above. In fact, Wallerian degeneration may proceed all the way to the nuclei gracilis and cuneatus at the cervico-medullary junction. The same comments apply to the spinothalamic tracts which carry pain and temperature sensation. There are several other afferent ascending spinal pathways that require to be restored, including the spinocerebellar tracts and Clarke's column, which provide inputs to the cerebellum and basal ganglia within the system of reflex connections responsible for coordinated movement. These ascending axonal fibers must also be persuaded to regrow through the lesions and make appropriate connections in order to 'cure' SCI.

\section{Central gray matter and propriospinal pathways}

Fortunately due to the overlapping territory of human dermatomes and myotomes, it will not be essential to replace the central gray matter at the level of injury gain a 'cure' for SCI. However, functional recovery of the propriospinal, short axonal, reflex network may be important. Restoration of this short axonal system capable of spanning the lesion may be useful as an alternative mechanism for recovery of function.

\section{The autonomic motor system}

The sympathetic pathways descending from the hypothalamus will also need to be properly reconnected to the intermedio-lateral gray matter in the thoracic cord. Sympathetic reflexes are important in maintaining blood pressure in relationship to posture and for the control of body temperature. Normal functioning of the bladder and bowel, especially of the sphincters and the sexual reflexes are also important considerations in recovery. Reconnection and programming of the parasympathetic nucleus of Onuf in the sacral cord is vital in this respect.

This is a brief resume of the neuroanatomy and neurophysiology of the human spinal cord. It is included in outline here so that the complexity of the requirements for a cure of SCI may be more fully appreciated by those whose expectations may be unrealistic. There is now great interest in the possibility of such a cure being produced by differentiating stem cells replacing the lost CNS cells and axons, a topic which is the focus of this review. The above description of the functional neuropathology of SCI serves the purpose of informing those scientists pursuing stem cell research of the neurological requirements for a cure.

\section{A brief review of current treatment of SCI}

It is useful to review what can be done now for the spinal-injured patient as a stepping stone toward a future 'cure'. Modern treatment for SCI rests on the 
foundation laid by the great pioneer Sir Ludwig Guttmann. It is relevant and appropriate to review his contribution and career highlights. In early life in Germany, Sir Ludwig trained as a neurosurgeon under Professor Foerster in Breslau from which university Sir Ludwig graduated. ${ }^{18,19}$

With the advent of National Socialism he left Germany for England in the 1930s but he did not follow his chosen vocation but was directed into pure research. However, with the advent of World War II he was assigned to look after the 'hopeless' cases of the spinal injured, where it was thought that he could do 'little harm'. Stoke-Mandeville Hospital was the outpost where the SCI patients were 'left to die' of urinary tract or respiratory infections away from the regular military hospitals at the time. However, Sir Ludwig set about treating these poorly regarded individuals vigorously and proceeded dramatically to turn around their 'hopeless' prognosis.

Sir Ludwig applied his knowledge of neurology and sterile surgical technique with great success and in so doing developed the principles of modern rehabilitation. Despite his training as a neurosurgeon he soon realized that laminectomy for supposed decompression of the spinal canal over the lesion was harmful in SCI and would often aggravate the neurological state of the patient. He thus became a strong advocate of conservative, that is, nonsurgical management of SCI. With some difficulty it became generally accepted that SCI was not a surgical emergency. Furthermore, any possible benefit of decompressive laminectomy was not supported by the neuropathology as the capacity of the spinal canal is automatically restored in most instances soon after injury and there are no space taking effects. Having been referred patients sent from other hospitals with advanced complications such as bedsores, infections and contractures, he insisted that all SCI patients be admitted immediately into his Spinal Unit. This policy was soon accepted worldwide commensurate with establishment of spinal units. ${ }^{20,21}$

The basis for conservative management of SCI was at first empirical. However, neuropathological studies that followed were able to confirm that there were few indications for intervention. Reduction of the vertebral deformity usually occurred spontaneously even in acute 'coroner's cases' who did not survive to reach hospital alive. Furthermore, neuropathological studies demonstrated the relative resistance of the spinal cord to the initial injury and that some continuity of spinal cord tissue across the lesion was common. This delicate lifeline could easily be compromized not just by poor transfer technique but simply by the effects of further surgery. Exposure and possible movement of the injured cord with hypoxia during surgery are detrimental factors. Furthermore, the inevitable trauma of the surgery itself and greater destabilization of the spine are other aggravating factors.

Energetic attention to the respiratory system with tracheostomy undertaken in high cervical lesions and robust physiotherapy kept the lungs clear. Similarly, urinary tract infections were avoided by aseptic catheterization. A team approach was developed to deal with every complication. The adverse systemic and nutritional effects of the injury were controlled by dietary management and the catabolic effect of trauma reduced. Autonomic dysfunction was controlled pharmacologically and blood pressure was maintained as best as possible. In all, $24 \mathrm{~h}$ care with regular turning and a supportive positive attitude underlay Sir Ludwig's therapeutic 'miracle'. In this way, an SCI patient was not condemned to severe morbidity and early death. Sir Ludwig's peers were astounded by these results. A death sentence had been converted to more or less normal life expectancy for the majority of patients many of whom returned to their homes and rejoined the workforce as productive citizens. Thus modern rehabilitation was born. A short time later, in Australia, Sir George Bedbrook achieved similar outstanding results. Sir George, an orthopedist, also advocated conservative management based on his own experience. His treatment was duly as vigorous in attacking the problem within a comprehensive medical regime and an army of therapists espousing the concept of 'total care' in the treatment of SCI. ${ }^{21,22}$

As may be expected, Sir Ludwig and Sir George became close friends. Both are recognized as the fathers of modern rehabilitation. Add to this Sir Ludwig's tremendous insight in recognizing sport for the disabled as a great factor in morale building in SCI and being generally beneficial. Thus, the Stoke Mandeville Games were established. These games evolved to become firstly the International Paraplegic Games and later the Paralympics now held every 4 years in conjunction with the Olympic Games.

It is true that spinal surgery following SCI is again in vogue, the justification being mainly economic. Rapid stabilization with internal fixation reduces time in hospital thus keeping down costs through early mobilization. However, it is not likely that either gentleman would agree with this trend. Although surgical technique has been refined in recent years being less invasive, it remains to be shown that the outcome of surgery is neurologically superior to conservative management in the long term.

More recently there has been a pharmacological addition to the treatment regime. Well-controlled clinical trials have shown that corticosteroids (methyl-prednisolone) administered in the acute phase conserves neurological function to a slight degree. Unfortunately, it is not certain that this benefit is sustained in the long term. The same may be said for 4-aminopyridine as a method for limiting axonal damage. However, both agents have been justified on scientific grounds and given the seriousness of the injury should be administered. Nevertheless, their universal application makes therapeutic trials of other drugs difficult to evaluate, for example, the value of GMI gangliosides or the testing of other agents. $^{23-25}$ 


\section{The search for a cure}

The potential of recent advances to provide a cure of SCI Animal experiments There are many animal models of SCI investigated over the years. The species used range from primitive vertebrates through to mice, rats, cats, sheep and primates. Injuries are produced by dropping weights on to the exposed spinal cord or by inflating balloons inserted extra- or intradurally and by other more sophisticated methods. ${ }^{26}$ The reaction to injury is investigated in these models and methods to limit the damage evaluated. These investigations include the study of the vascular and inflammatory reactions, release of cytokines, upregulation of primitive genes, trophic factors, their receptors and cell death due to necrosis and apoptosis. The object of these experiments is to provide an understanding of the process responsible for the dissolution of the tissue elements of the spinal cord at the level of injury, and to search for methods designed to arrest this loss. The vascular reaction, which is inherent in the inflammatory process, has been recognized as the main cause of such 'secondary damage' and is attributed to cytokines and products of leucocytes. An example of the focus on the inflammatory reaction is the use of CMIOI in mice, which has been shown to preserve posttrauma conductivity. ${ }^{27}$ Free radicals have been postulated to aggravate the lesion so that scavengers of free radicals have been used with some benefit. ${ }^{28}$ The opiod antagonist naloxone has also been applied with apparent success. ${ }^{29}$ More sophisticated investigations explore the molecular events with same objective. ${ }^{30}$ Many other agents have been tested for their neuroprotective effect and of these methylprednisolone and 4-aminopyridine have reached the clinic as mentioned above. ${ }^{23,24}$

Beyond the acute phase following injury in which the approach has been to limit the damage, much attention has been given by basic scientists to the inability of the adult CNS to repair itself, a universal occurrence in mammals, in contrast to more primitive species. For instance, in the chick CNS regeneration occurs as it does in the embryo of other species. Even more tantalizing is the fact that CNS regeneration is spontaneous in adult fish and lizards following injury. These species have also been extensively investigated because the neurobiological information obtained may hold the key to regeneration in mammals. ${ }^{31-33}$

Fawcett ${ }^{34}$ has analyzed the neurobiological basis for the absence of central axonal regeneration and has considered what may be done to overcome this deficiency. He reviews the successful experiments in which some central axonal regeneration has been achieved as follows. The first group of experiments encompass the inhibitory molecules that block central regeneration. The primary member of such inhibitory molecules is derived from myelin and is known as N1250. Schwab and his team ${ }^{35}$ developed an antibody to this compound naming it IN-1 and applying it with success producing about $1 \mathrm{~cm}$ of central regeneration in experimental SCI. Another myelin-associated inhibitory molecule which has been identified is MAG for which no blocking antibodies can be produced. The same applies to the proteoglycans related to astrocyte and oligodendrocyte precursors, against which no blocking mechanism has been discovered. ${ }^{34}$

Grafts and transplants Going further neuroscientists have explored the possibility of replacing the lost tissue with embryonic and fetal grafts. These experiments have had variable results, some quite encouraging. ${ }^{36,37}$ The greatest advance in the field of grafting was made by Albert Agayo and co-workers, ${ }^{38,39}$ who transplanted peripheral nerve from the optic nerve to the tectum and found that peripheral Schwann cell myelin enabled central axonal regeneration to occur. The phenomenon has been intensely investigated since. ${ }^{40}$ Thus, it became evident that Schwann cells are permissive of central regeneration and many experiments have since taken place based on this principle. Outstanding among these is the work of Ramon et al. ${ }^{41}$ These workers have obtained a considerable amount of central axonal regeneration by using a bridge containing Schwann cells supplemented by olfactory ensheathing cells (OEC). The OEC have the ability to migrate into the neighboring tissues enhancing regeneration, whereas the Schwann do not migrate out. It is reported that in each of these animal experiments some functional recovery occurs. Other experimenters employ the trophic factors FGF1, added to the peripheral nerve grafts with successful central regeneration and return of function. ${ }^{42}$ Macrophages have also been shown to provide a suitable environment for central axonal regeneration. ${ }^{43}$ The first experiments showing this effect involved the retina and many others have since followed. Fetal transplants have also been used in the brain of animals with variable results. More success has been achieved in grafting the hippocampus. ${ }^{44}$ Fetal transplants have also been implanted into the injured rat spinal cord where they migrate and differentiate. ${ }^{45}$ However, these CNS grafts are site specific which is a limiting factor.

Incidentally, human fetal spinal cord has also been used in the hope of arresting post traumatic syringomyelia and related pain rather than to induce regeneration of central axons. ${ }^{46}$ At a later stage following injury investigations have been directed to reducing gliosis and collagenous fibrosis which are believed by some to obstruct regeneration mechanically. ${ }^{47,48}$ In recent times, regeneration research has taken a new direction in finding human application with much attention being now given to exploring multipotential stem cells in bringing about CNS repair and regeneration. This topic will now be discussed.

\section{Cellular therapy}

Further to the grafting of fetal tissues, greater success in CNS regeneration is anticipated using clones of single cells that have the potential to differentiate into neurons, with axons or glia. Most specialized tissues of the human body are now known to house 'reserve' cells, 
which have the ability to differentiate and replace lost cells. These resting cells which are capable of multiplying and differentiating, are variously known as 'primitive' cells, for example, in the bone marrow or as 'reserve' cells in the lung and skin and as 'satellite' cells in muscle. Similar immature cells have even been identified in the brain where they line the ventricles and central spinal canal. It is hoped that the use of undifferentiated cells will bring about $\mathrm{CNS}$ repair but there are also disadvantages in cell therapy. A recurring difficulty is immunological rejection by the host. Although much has become known about histocompatability factors and pharmacological immunosuppression has improved graft acceptance rejection remains a serious limiting factor in this field. One reason for turning to host-derived multipotential cells is to avoid such tissue rejection. ${ }^{49}$

Stem cells There are three types of host-derived cells that have the potential to differentiate into neural tissues, neurons and/or glia. These are primitive bone marrow (BM) cells, which have the ability to differentiate into neurons, ${ }^{50}$ olfactory ensheathing cells (OEC) and neural precursor cells (NPC), which lie within the matrix lining the cerebral ventricles, central canal of the spinal cord and in the dentate gyrus of the hippocampus. Because of their ability to differentiate and replace lost tissues, the undifferentiated resting cells in adult tissues have been referred to as a type of stem cell. This term, however, has its origin in embryology referring to primitive cells that give rise to progeny, which grow to specialize eventually into the adult forms. The initial promise that self-derived adult stem cells would be suited to widespread application in tissue repair and regeneration and thus avoiding the immunological rejection is not likely to be fulfilled. Adult 'stem cells' merely fuse with existing cell when forming new tissue and their ability to multiply is not unlimited as for embryonic stem cells. ${ }^{51}$ Emergent ethical barriers confront the use of human embryonic stem cells (ESC), for example, Presidents Bush's policy of allowing only the use of existing embryonic cell lines. This is not a satisfactory solution because it is only ESC which have the full potential of bringing about CNS repair. The Australian policy is more enlightened since it is permissible to use surplus embryos from IVF clinics.

Stem cells have gained favor as a means to cure disease because of their ability to produce fully differentiated progeny. Classically when the stem cell divides one daughter cell goes on to differentiate and the other remains in a primitive pluripotential state retaining the ability to multiply indefinitely. Along with these morphological observations much has also become known of the molecular mechanisms and the associated genes which govern differentiation. Thus, phenotypic properties and the genes regulating CNS plasticity may also be identified in this way. It is this combined knowledge which holds the key to healing the CNS. Proteomics, which identifies the many proteins present at different stages of development and hence the genes expressed in differentiation, is an additional powerful tool in this research. ${ }^{52}$

In addition to their ability to replace lost or degenerate tissues genetically engineered transgenic ESC may also serve as vectors for the delivery of trophic factors, neurotransmitters, cytokines or even therapeutic viruses. ${ }^{52}$ Further to their ability to multiply and produce new tissues, ESC can also migrate and integrate with existing tissues. Furthermore, they have been found to express genes that encourage repair and more surprisingly may induce degenerate cells per se to recover. This property also holds promise in neurology for those disorders characterized by neuronal degeneration such as Alzheimer's and motor neuron diseases. ${ }^{53}$

The inner cell mass of the blastula is the usual source of ESC. These cells have the ability to differentiate into any form of adult tissue but it should be noted that in the early mouse experiments teratoma formation sometimes developed. ESC are by far the favored source of stem cells for the repair of neurological tissue including the spinal cord. One of the great attributes of ESC is that initially they are immune tolerant. This property has been found to be due to their expression of the Fas ligand. ${ }^{52}$ It should be recognized that ESC do become immunogenic when they mature. This limitation of their use undermines one of ESC stated advantages over other cells. However, immune rejection may be reduced in the same way as for other non-self-tissues by the administration of cyclosporin and other immunosuppressives. MHC matching of donor and recipient may also be of value as it is for transplants generally. As stated above it is unfortunate that the complex and difficult ethical issues have delayed human ESC experiments, which may prove their safety and value in treatment of all diseases not just SCI. ${ }^{52}$

In humans there is suggestive evidence that new neurons may also be generated by the NPC of the hippocampus. Early experiments show that these cells have the ability to grow into glia or neurons depending on their site of origin. Of great interest is the report of functional changes resulting from new connections made by the newly differentiated NPC. ${ }^{51}$ Embryonic neural stem cells (NSC) also hold promise for healing neurological diseases. NSC are derived from the neuroepithelium of the embryo. During development, these cells migrate from the matrix zone lining the primitive ventricles and give rise to populations of astrocytes oligodendrocytes and neurons all of which express nestin. ${ }^{54}$ A limitation of NSC is that they are known to be subject to the Hayflick limit, that is, 40 cell divisions in vitro. As such they do not have the property of unlimited growth characteristic of ESC. ${ }^{51,55}$ Nevertheless, NSC are the stem cells most often used so far in neurological experiments (see below).

\section{Stem cell experiments on animal models of neurological} disease

The most successful experiments have been on the wellknown neurological mutant mice. For instance, NSC 
introduced into newborn 'meander tail' mice, which are deficient in cerebellar granule cells are able to migrate into the neuron-deficient sites and replace a proportion of the lost cells. ${ }^{56,57}$

Another neurological mutant is the 'reeler' mouse which shows aberrant neuronal migration during development. NSC implanted at birth have been shown to improve the cytoarchitecture of the developing cerebral cortex. This change occurred presumably by the NSC replacing the extracellular matrix signal normally provided by the gene 'reelin' which is mutated in the 'reeler mouse'. 56

A further model of neurological disease is the 'shiverer' mouse, which lacks myelin basic protein (MBP). In experiments, NSC were shown to posses the ability to migrate widely in these mice. They were shown to differentiate into new oligodendrocytes, which contained the missing MBP and thus formed normal myelin. Also quite markable in these experiments is the reported functional improvement observed in the treated mice. ${ }^{58}$ Implanted NSC have also been used to replace metabolic disorders in mutant mice. An example of this is in 'twitcher' mouse which has the equivalent of human Krabbe's disease (globoid cell leukodystrophy). In this case, the newly differentiated neurons derived from the NSC produce 'galactocerebroside alpha'. The treatment corrects the defect morphologically but unfortunately no functional improvement is evident from these experiments. ${ }^{56}$ NSC have been used in other animal models of human neurological disorders with variable outcomes. Examples are multiple sclerosis, Parkinson's disease and motor neuron disease (ALS). They have also been applied to experimental stroke. $^{53}$

In the experimental SCI model of contused adult rat spinal cord, NSC develop into neurons that may migrate long distances rostrally and caudally. In these experiments, the treated rats are said to show functional improvement. Many such experiments have been undertaken and are generally well known. ${ }^{53}$ Concerning SCI in animal experiments recently an inhibitor of cAMP, rolipram, has been shown to enhance central regeneration in rats when combined with Schwann cell implants. It is reported that 'this treatment further enhances axonal sparing and myelination, promotes growth of seratonergic fibers into and beyond grafts and significantly improves locomotion'. ${ }^{59}$ Another recent report describes the success of umbilical cord blood stem cells in improving experimental SCI in rats. ${ }^{60}$

Olfactory ensheathing cells (OEC) have also been used with considerable success in gaining axonal regeneration in experimental SCI. These cells, which continuously replace the nasal epithelial cells, have the ability to differentiate into neurons and create a permissive environment for axonal regeneration. They also have the advantage of being able to migrate into the surrounding tissues. ${ }^{61-64}$ ESC have also been shown to have the ability to differentiate into oligodendrocytes and myelinate naked axons in culture. The outcome in animal models of SCI is improved by the use of suitable polymer scaffolds within the lesion site designed to provide a framework for the regenerating axons. ${ }^{65}$

Raisman $^{44}$ addresses the issue of CNS regeneration from a slightly different perspective. He envisages inhibitory molecules to be part of a guidance system for central axons during development but aborting axonal regeneration in the adult, that is, becoming inhibitory when the axon leaves its correct pathway. He points out that when a central nerve fiber is cut it sprouts vigorously but such growth is soon aborted. Recognizing this capability he suggested bridging the gap in experimental injuries with transplanted cells which will provide the guidance molecules. He and his team have chosen OEC for this purpose. ${ }^{44}$ As an example, cultured OEC have been transplanted into unilateral lesions of the cervical corticospinal tract in adult rats. Such grafted cells allow regenerating central axons to penetrate the bridge and grow into the distal stump. Interestingly, the OEC culture was found to develop into Schwann and fibroblast-like cells, which were found to ensheath the regenerating axons. These nerve fibers were seen to continue in the graft and form their normal auborizations with their target neurons. When these new axons left the graft they were covered by oligodendrocytes. Even more encouraging is the fact that these reconstructed spinal rats were able to recover function of a learned task. ${ }^{44}$

\section{Preliminary stem cell experiments in humans with neurological diseases}

In the recent past, there have been two main lines of research in humans using stem cells. These are for the treatment of Parkinson's disease and in SCI. In Parkinson's disease 'expanded neurological precursors' (ENP) have been implanted into the substantia nigra with success in most reports although results have been variable. ${ }^{66}$ Going further, because of the serious nature and clinical urgency it is not surprizing that there are several stem cell experiments in progress for human SCI in different parts of the world. The International Campaign for Cures of Spinal Cord Injury Paralysis (ICCP) held a Workshop in Vancouver early in 2004 in order to assess progress. In these experiments, ESC, OEC and macrophages have been introduced in order to repair the human spinal cord. ${ }^{67}$ The final outcome of these human experiments will be closely examined when the results are published. At present, some reservation is expressed concerning the timing of such human trials since a reliable stem cell treatment protocol has not yet been developed in an animal model nor its safety proven (see below).

\section{The future}

It is true that stem cells offer hope that a cure for human SCI will be found based on this technology. In order to reach this goal there are a number of features of the neuropathology of SCI which must be taken into account. The lesion site is to be replaced with CNS 
tissue, the long tracts restored and integrated with their proper neurophysiological connections. It may not be absolutely essential to reconstitute the central gray matter of the lesion because of the overlapping segmental supply of dermatomes and myotomes. It is the interruption of the long tracts which determines the neurological status of the patient. When and if regenerated these long axons are to be physiologically connected to the CNS centers above the lesion and to the neuronal pool below. The centers themselves may require 'reprogramming' so that the reflexes, neurotransmitters and neuroendocrines, which determine normal function, return to normal.

Voluntary and involuntary (extrapyramidal) motor functions, conscious sensation and autonomic activity each are to be returned to normal for a cure to be considered successful. Although a complete return of these neurological functions is desirable, lesser degrees recovery may prove to be very helpful in rehabilitation. The reacquisition of motor control sufficient for independent ambulation and the return of some sensation and socially adequate autonomic functions would be a major advance. A limited amount of recovery would be a boon to many thousands of persons the world over. In this respect, a guide to the minimum number of axons required for partial recovery has been explored in human material using the principle of clinico-pathological correlation details of which are given above. 5,9

\section{First principles}

The first step is to maintain the SCI patient in an optimal condition medically by means of modern treatment and rehabilitation. ${ }^{68}$ It is logical that in order to obtain the greatest benefit from any stem cell therapy which may be developed in the future, the SCI patient should be maintained in the best physical, psychological and emotional state possible. Apart from standard treatment including bladder and bowel control with any infection treated immediately, regular physical therapy preferably with FES is recommended. This regime may be supplemented by extra-dural electrical stimulation and other methods of restorative neurology if available. ${ }^{69}$ Electrical stimulation is known to facilitate well-being in incomplete SCI. ${ }^{70}$ It is also important to avoid pressure palsies of peripheral nerves so that the skeletal muscles do not undergo atrophy.

Clinical experience shows that regular sensory stimulation through the skin has the effect of keeping retained neurological functions and returning reflex nearer to their normal physiological state. It also has the effect of reducing unwanted 'spasms'. Furthermore, it seems that very intensive sensory input, for example, constant massage may be responsible for some rare instances of late recovery of motor or sensory function such as that reported by $\mathrm{Mr}$ Christopher Reeve. This phenomenon also underlines the importance of recognizing the discomplete SCI individuals who may benefit from treatment within the repertoire of restorative neurol- ogy. ${ }^{69}$ This standard clinical regime for SCI is the treatment platform from which the stem cell therapy when developed may be mounted.

The potential of stem cell therapy to cure human SCI It should be recognized that stem cell treatment is by its nature invasive so that there are certain risks involved. Apart from the generally applicable hazards of surgery, there is the possibility of introducing harmful viruses contained with the transplants into the body or malignant transformation of the introduced cells. ${ }^{71}$ It would be a tragedy if the patients neurological state and degree of disability was made worse so that a note of caution is advisable.

It is not known at present which type of stem cell will ultimately be best and each will need to be thoroughly evaluated in an animal model before it can reasonably applied to human SCI despite the clinical and social urgency to find a cure. As mentioned above, hostderived stem cells from either bone marrow or the patients own Schwann cells and OEC would be preferred to avoid immunological rejection. However, it has been shown experimentally that such cells do not possess the best potential for growth and differentiation. Human embryonic stem cells have much greater potential but they are necessarily foreign. When undifferentiated ESC are tolerated immunologically, but they become antigenic when mature thus rejection is not altogether avoided and immunosuppressive treatment with its hazards may be required. At present due to legal and ethical constraints, human ESC are not readily available and those in store in the USA are known to be gradually deteriorating. ${ }^{52}$

It is strongly recommended that any projected stem cell therapy be proven for safety and effectiveness in experimental animals prior to human application. It will need to be shown that ESC are capable of providing the environment required for axonal regeneration and be able to differentiate into neurons in the experimental spinal model. Another possibility is that it may not be necessary for the ESC to replace the lost tissue fully. It is possible that they will engender self-repair by fusion with host cells. In animal experiments, it has been shown that introduced stem cells may not grow into neurons and axons but in some way provide an environment which causes the host tissue to recover from degenerations. ${ }^{56}$ Putting these important considerations aside how would ESC bring about a cure? An analysis of requirements to reach this objective follows.

\section{Treatment plan for human SCI}

Fawcett ${ }^{34}$ has laid out a treatment plan for applying the discoveries in neurobiology, which bring about axonal regeneration in human SCI. This protocol was developed before stem cell technology became available. However, these advances may be readily added. The first step would be the delicate surgical removal of astrocytic and collagenous scar tissue and the unwanted nerve root 
regenerations but carefully preserving any residual central axons traversing the lesion. This procedure will require expert microsurgery painstakingly undertaken to avoid damaging residual spinal cord parenchyma with its axonal fibers. Fawcett ${ }^{34}$ suggests that a bridge of Schwann cells and OEC derived from the patient's own tissue and expanded in culture be applied to the lesion. Axonal regeneration may be stimulated by trophic factors together with blocking molecules infused through an indwelling catheter. The local environment would be made more permissive for axonal regeneration by neutralizing inhibitory factors with blocking antibodies such as IN-1.

ESC could be added to this regime bringing it up to date. Rolipram which also enhances regeneration, may also be included. Before introducing ESC it will need to be adequately shown in an experimental model that they produce the expected outcome. That is, the ESC stimulating axonal regeneration by fusing with host cells and/or provide new axons which connect with the ends of the fibers of the long tracts. The terminal points of disrupted afferent and efferent axons destroyed by the injury will need to be regenerated. Such capability may be enhanced by molecular genetic manipulation whereby the introduced stem cells may be engineered to express trophic factors as has been done experimentally. ${ }^{72,73}$ These axonal endings may be some distance away due to Wallerian degeneration. It will be necessary for these stimulating factors to be transported to the respective cell bodies, Betz cells above, posterior horn neurons and root ganglia below the lesion site in order to bring this about.

As mentioned above, to return the neurophysiological function of the spinal cord to normal it is the residual long axons that need to be regenerated. However, the distance to be traversed is far greater than any axonal regrowth so far achieved in an animal which is not more than a centimeter or so. An alternative to long axonal growth would be to create a multisynaptic pathway, the introduced stem cells differentiating into neurons and forming a network. This network will need to reach from the ends of the severed cortico-spinal axons to the anterior horn motor neurons below. For the sensory systems, the medulla cerebellum and thalamus above will need to be connected. Whether such a multisynaptic pathway would be functionally useful is problematic as it will be inherently slow conducting, for example, reflexes will be out of synchrony. On the other hand, some optimism is provided by the remarkable attribute of plasticity which may compensate for this deficiency. To recover ambulation, the central pattern generator in the lumber spinal cord will need to be reconnected and rendered physiologically functional. The same considerations apply to the efferent extrapyramidal and autonomic systems.

In this context, it is at least somewhat reassuring that our own post-mortem studies have shown that Betz cells are not lost even in long-standing anatomically complete SCI. It has also been demonstrated that the motor neurons are retained in the segments below the lesion and are not lost transneuronally but are preserved in normal number. ${ }^{74}$ It is also helpful that the peripheral nerves are generally intact in SCI so that the muscles are normally innervated and have not undergone severe atrophy. Because the lesion is of upper motor neuron type the skeletal muscles do not undergo atrophy. However, there is a change in the proportion of histochemical muscle fiber types, type 2 fibers becoming more numerous in chronic SCI. Red type 1 (tonic) muscle fibers are converted to the type 2 (phasic rapidly contracting) white muscle fibers. Type 1 fibers are oxidative and are more concerned with posture and tone. It is probable that with recovery of voluntary motor control, the proportion of fiber types will return more toward the normal distribution. Retraining with FES may assist this process. To complete the cure the regenerated central axons will need to reach their correct destinations making synaptic connections appropriately and discharging the right amount and type of transmitter, excitory or inhibitory and be modulated at the correct thresholds. In addition, a relearning and retraining educational regime will be necessary.

All of this appears to be overwhelmingly difficult and unlikely to occur given the complexity of the CNS. However, considerable encouragement is offered by noting the quite remarkable way in which with a little help the CNS can recover lost functions. An example is the way in which Levodopa given orally reaches the substantia nigra and compensates for the loss of dopaminergic neurons in Parkinson's disease with resulting great clinical benefit. In the same vein, it is surprising that rather primitive electrical stimulation of basal ganglia may also assist Parkinson's patients by reducing tremor and improving gait. Another even more spectacular example of how with artificial input the CNS may adapt and recover function is provided by the great success of the bionic ear developed by Graeme Clark et al. ${ }^{75}$ Similar but less outstanding results have been achieved for the substitution of vision using tactile stimulation. $^{76}$

Another reason for optimism is the well-known ability of the CNS to respond to external and internal stimuli by changing its synaptic connections by means of plasticity. The relearning of skills and language after stroke or head injury is due to this phenomenon, best seen in the young in whom language centers may even be relocated to another topographic site in the brain. It is anticipated that plasticity will also contribute to the recovery of CNS functions following axonal regeneration which may be induced by stem cell and adjuvant therapy in the future. It is well-known neurophysiologically that the organization of the CNS responds to everyday sensory inputs and is constantly changing. Reflexes are driven in this way and greater or lesser numbers of neurons are recruited as necessary, thus the creation of neurological 'centers'. It is evident that the brain functions as a whole with one part taking over readily from another. Therefore, it is the property of plasticity which contributes to the hope that a cure of SCI will result even if only crude neuronal reconnection 
are achieved. Well-planned experiments in animal models will be needed to reach each of these objectives step-by-step before human studies begin. Some are in progress and a greater refinement of the neurobiology concerned is well underway, ${ }^{77-79}$ AMD will serve to enhance a treatment protocol in an experimental animal.

\section{Discussion}

Injuries of the spinal cord are common and devastating. The human suffering and economic burden posed is gargantuan and no place on earth is exempt. In early times SCI was the uncommon result of falls, sporting accidents or due to battle trauma. Industrialization and motorized transport have created this modern catastrophe. Eleven thousand new SCI occur in USA annually where it is estimated there are 250000 patients with such injuries. Apart from the human tragedy the average lifetime cost in the order of over US\$1 m.

The high degree of urgency in finding a cure for SCI is a reflection of the large numbers affected and the devastating nature of SCI. Unfortunately, very little benefit has been derived clinically from basic research in regeneration so far. Some useful experimental models have been produced but to the present generally this work has been unrewarding in terms of improved clinical treatments. Molecular biology has led to the recognition of factors, which may inhibit or enhance axonal regeneration and of cytokines causing 'secondary damage'. Also recently recognized are the 'early genes' expressed by the injury, trophic factors and guidance molecules, for example, the EPH family of genes. With this knowledge, some experiments involving transplantation and grafting have been moderately successful. Leading this field was the demonstration by Albert Agayo $^{39}$ and his team ${ }^{40}$ who showed that grafts of peripheral Schwann cell myelin made central axonal regeneration possible. Unfortunately, this discovery has not led to an effective treatment for SCI.

The existence of reserve cells in tissues other than bone marrow and hematological stem cells have been known for many years. Their ability to replace lost cells has been utilized in therapy mainly in the treatment of leukemia and some metabolic diseases.

Indeed, toward the end of the last century myoblast transplants were the subject of intense research in the hope of producing a cure for muscular dystrophy. Regrettably this proved to be unsuccessful.

More recently the same intentions have been transposed to a wider range of conditions including SCI because of the potential for embryonic stem cells to differentiate in accordance with their host environment. As described above this optimism is supported by success with neural fetal transplants for the treatment of degenerative neurological disease in animals and to some extent in the human notably Parkinson's disease. As is case with most watershed developments in medical research a massive amount of discussion and conjecture has arisen both in the lay and medical literature about stem cells, which have been touted as the answer for many incurable disorders ranging from Alzheimer's disease and other neurodegenerations, through to head injuries and SCI. The properties of the various types of stem cells and their therapeutic potential is reviewed above and a more realistic appraisal of their value provided.

\section{Conclusion}

In the context of finding a cure for SCI the first and foremost requirement is an in-depth knowledge of the disorder in neuropathological terms with the complexity of the spinal cord appreciated. To bring about central axonal regeneration and restoration of normal function is a formidable task and those neurobiologists planning experiments need to keep the details of the neuropathology of SCI in mind. There is much work in progress exploring the possibility of stem cells being able to cure SCI. It is true that ESC hold great promise in leading to an eventual cure of SCI. A comprehensive approach in methodology taking the neuropathology and complex neurophysiology into account is called for. Unfortunately, some human experiments have commenced prior to a successful treatment plan being developed in an experimental animal model of SCI. It is strongly recommended that before human stem cell trials begin, the treatment regime should be well worked out experimentally beforehand. The establishment of a large center for stem cell research at Cambridge University is a very positive development which will greatly assist in these endeavours. Fortunately, the United Kingdom has adopted a more flexible ethical policy so that the new Cambridge Stem Cell Institute will have a reasonable opportunity to produce the promised outcomes in medicine and biology.

\section{Acknowledgements}

The support of the Neurotrauma Research Program (NRP) of the Western Australian Government which provided a grant through the WA Institute for Medical Research for the study of the neuropathology of human SCI is gratefully acknowledged.

\section{References}

1 Hughes JT. Pathology of the spinal cord. Major Problems Pathol 1978; 6: 1-257.

2 Kakulas BA. The applied neuropathology of human spinal cord injury. Scientific Review. Spinal Cord 1999; 37: 79-88.

3 Kakulas BA, Taylor JT. Pathology of injuries of the vertebral column and spinal cord. In: Vinken PJ, Bruyn BW, Klauwens HL, Frankel HL (eds). Handbook of Clinical Neurology. Elsevier Sciences Publishers: Amsterdam, 1992 pp 21-51.

4 Willen J, Gaekwad UG, Kakulas BA. Burst fractures in the thoracic and lumbar spine - a cliniconeuropathological analysis. Spine 1989; 14: 1316-1323.

5 Kakulas BA. A review of the neuropathology of human spinal cord injury with emphasis on special features. J Spinal Cord Med 1999; 22: 119-124. 
6 Kakulas BA, Lorimer RL, Gubbay AD. White matter changes in human spinal cord injury. In: Stalberg E, Sharma HR, Olsson Y (eds). Spinal Cord Monitoring: Basic Principles, Regeneration, Pathophysiology and Clinical Aspects. Springer-Verlag Publishers: Wein, New York, 1998 pp 395-407.

7 Bunge RP, Puckett WR, Becerra JL, Marcillo A, Quencer RM. Observations on the pathology of human spinal cord injury. A review and classification of 22 new cases with details from a case of chronic cord compression with extensive focal demyelination. In: Seil FJ (ed). Advances in Neurology, Vol. 59. Raven Press: New York, 1993 pp 75-89.

8 Dimitrijevic MR. Residual motor functions in spinal cord injury. In: Waxman SG (ed). Functional Recovery in Neurological Disease. Raven Press: New York, 1988 pp 139-155.

9 Kaelan C. Neuropathology and clinical neurobiology of human spinal cord injury (SCI): quantitation of tissue elements in human SCI and the comparison of experimental animal SCI and human SCI. PhD Thesis, University of Western Australia, 1993.

10 Wolman L. The disturbance of circulation in traumatic paraplegia in acute and late stages: a pathological study. Paraplegia 1965; 2: 213-226.

11 Tator C. Update on the pathophysiology and pathology of acute spinal cord injury. Brain Pathol 1995; 5: 407-413.

12 Tator $\mathrm{CH}$. Biology of neurological recovery and functional restoration after spinal cord injury. Neurosurgery 1998; 42: 696-707.

13 Curt A, Dietz V. Electrophysiological recordings in patients with spinal cord injury: significance for predicting outcome. Scientific Review. Spinal Cord 1999; 37: 157-165.

14 Ito T, Oyanagi K, Wakabayashi K, Ikuta F. Traumatic spinal cord injury: a neuropathological study on the longitudinal spreading of the lesions. Acta Neuropathol 1997; 93: 13-18.

15 Buss A et al. Gradual loss of myelin and formation of an astrocytic scar during Wallerian degeneration in the human spinal cord. Brain 2004; 127: 34-44.

16 Grillner S. Neural networks for vertebrate locomotion. The motions animals use to swim, run and fly are controlled by specialized neural networks. For a jawless fish known as the lamprey, the circuitry has been worked out. Sci Am 1996; 274: 48-53.

17 Pinter MM, Dimitrijevic MR. Gait after spinal cord injury and the central pattern generator for locomotion. Spinal Cord 1999; 37: 531-537.

18 Frankel H. 100 years after his birth Guttmann's message lives on. Spinal Cord 1999; 37: 461-462.

19 Harris P. Professor Sir Ludwig Guttmann, FRS. Spinal Cord 1999; 37: 463-464.

20 Kakulas BA, Bedbrook GM. Pathology of injuries of the verteral column with emphasis on the macroscopical aspects. In: Vinken PJ, Bruyn GW (eds) in collaboration with Braakman R, Klawans HL Jr (Assoc Ed). Handbook of Clinical Neurology. Injures of the Spine and Spinal Cord Part I, Vol 25. North-Holland Publishing Company: Amersterdam, American Elsevier Publishing Co Inc: New York, 1976 pp 27-42.

21 Bedbrook G. The Care and Management of Spinal Cord Injuries. Springer-Verlag: New York, 1981.

22 Kakulas BA. Problems and solutions in the rehabilitation of patients with progressive muscular dystrophy. Scand $J$ Rehab Med 1999; 39(Suppl): 23-37.
23 Bracken MB et al. A randomized, controlled trial of methylprednisolone or naloxone in the treatment of acute spinal cord injury. $N$ Engl J Med 1990; 322: 1405-1411.

24 Potter PJ, Hayes KC, Hsieh JTC, Delaney GA, Segal JL. Sustained improvements in neurological function in spinal cord injured patients treated with oral 4-aminopyridine: three cases. Spinal Cord 1998; 36: 147-155.

25 Geisler FH. Clinical trials of pharmacotherapy for spinal cord injury. Ann NY Acad Sci 1998; 845: 374-381.

26 Stokes BT, Jakeman LB. Experimental modeling of human spinal cord injury: a model that crosses the species barrier and mimics the spectrum of human cytopathology. Spinal Cord 2002; 40: 101-109.

27 Wamil AW, Wamil BD, Hellerqvist CG. CM101-mediated recovery of walking ability in adult mice paralyzed by spinal cord injury. Proc Natl Acad Sci USA 1998; 95: 13188-13193.

$28 \mathrm{Li} \mathrm{GL}$, Farooque M, Holtz A, Olsson Y. Effects of alpha-phenyl- $N$-tert-butyl nitrone (PBN) on compression injury of rat spinal cord. Free Radical Res 1997; 27: 187-196.

29 Winkler T, Sharma HS, Stalberg E, Olsson Y, Nyberg F. Naloxone reduces alterations in evoked potentials and edema in trauma to the rat spinal cord. Acta Neurochirurgica (Suppl) 1994; 60: 511-515.

30 Brook GA et al. Attempted endogenous tissue repair following experimental spinal cod injury in the rat: involvement of cell adhesion molecules L1 and NCAM? Eur J Neurosci 2000; 12: 3224-3238.

31 Hasan SJ et al. Functional repair of transacted spinal cord in embryonic chick. Restorative Neurol Neurosci 1991; 2: 137-154.

32 Keirstead HS, Hasan SJ, Muir GD, Steeves JD. Suppression of the onset of myelination extends the permissive period for the functional repair of embryonic spinal cord. Proc Natl Acad Sci USA 1992; 89: 11664-11668.

33 Schwab ME, Brosamle C. Regeneration of lesioned corticospinal tract fibers in the adult rat spinal cord under experimental conditions. Spinal Cord 1997; 35: 469-473.

34 Fawcett JW. Spinal cord repair: from experimental models to human application. Spinal Cord 1998; 36: 811-817.

35 Bregman BS et al. Recovery from spinal cord injury mediated by antibodies to neurite growth inhibitors. Nature 1995; 378: 498-502.

36 Tessler A et al. Embryonic spinal cord transplants enhance locomotor performance in spinalized newborn rats. $A d v$ Neurol 1997; 72: 291-303.

37 Diener PS, Bregman BS. Fetal spinal cord transplants support growth of supraspinal and segmental projections after cervical spinal cord hemisection in the neonatal rat. J Neurosci 1998; 18: 779-793.

38 Aguayo AJ, Bray GM, Carter DA, Villegas-Perez MP, Vidal-Sanz M. Regrowth and connectivity of injured central nervous system axons in adult rodents. Acta Neurobiol Exp 1990; 50: 381-389.

39 Bray GM, Villegas-Perez MP, Vidal-Sanz M, Carter DA, Aguayo AJ. Neuronal and non-neuronal influences on retinal ganglion cell survival, axonal regrowth, and connectivity after axotomy. Ann NY Acad of Sci 1991; 633: $214-228$.

40 Harvey AR, Plant GW. Schwann cells and fetal tectal tisse co-grated to the midbrain of newborn rats: fate of Schwann cells and their influence on host retinal innervation of grafts. Exp Neurol 1995; 134: 179-191. 
41 Ramon CA, Plant GW, Avila J, Bunge MB. Long-distance axonal regeneration in the transected adult rat spinal cord is promoted by olfactory ensheathing glia transplants. J Neurosci 1998; 18: 3803-3815.

42 Cheng H, Cao YH, Olson L. Spinal-cord repair in adult paraplegic rats - partial restoration of hind-limb function. Science 1996; 273: 510-513.

43 Rapalino $\mathrm{O}$ et al. Implantation of stimulated homologous macrophages results in partial recovery of paraplegic rats. Nat Med 1998; 4: 814-821.

44 Raisman G. A promising therapeutic approach to spinal cord repair (editorial). J R Soc Med 2003; 96: 259-261.

$45 \mathrm{Wu} \mathrm{S}$ et al. Migration, integration, and differentiation of hippocampus-derived neurosphere cells after transplantation into injured rat spinal cord. Neurosci Lett 2001; 312: 173-176.

46 Falci $\mathrm{S}$ et al. Obliteration of a post-traumatic spinal cord cyst with solid human embryonic spinal cord grafts: first clinical attempt. J Neurotrauma 1997; 14: 875-884.

47 Morin-Richaud C, Feldblum S, Privat A. Astrocytes and oligodendrocytes reactions after a total section of the rat spinal cord. Brain Res 1998; 783: 85-101.

48 Privat A, Chauvet N, Gimenez Y, Ribotta M. Neuronal regeneration and the glial barrier. Rev Neurol 1997; 153: 515-520.

49 Wagers AJ, Christensen JL, Weissman IL. Cell fate determination from stem cells. Gene Therapy 2002; 9: 606-612.

50 Hess DC, Hill WD, Carroll JE, Borlongan CV. Do bone marrow cells generate neurons? Arch Neurol 2004; 61: 483-485.

51 Barker RA, Jain M, Armstrong RJE, Caldwell MA. Editorial: stem cells and neurological disease. I Neurol Neurosurg Psychiatry 2003; 74: 553-557.

52 Lemoine NR. The power to deliver: stem cells in gene therapy. Gene Therapy 2002; 9: 603-605.

53 Tai Y-T, Svendsen CN. Stem cells as a potential treatment of neurological disorders. Curr Opin Pharmacol 2004; 4: 98-104.

54 Alvarez-Buylla A, Garcia-Verdugo JM, Tramontin AD. A unified hypothesis on the lineage of neural stem cells. Nat Rev Neurosci 2001; 2: 287-293.

55 Hayflick L. Mortality and immortality at the cellular level. A review. Biochemistry (Mosc) 1997; 62: 1180-1190.

56 Park KI et al. Global gene and cell replacement strategies via stem cells. Gene Therapy 2002; 9: 613-624.

57 Rosario CM et al. Differentiation of engrafted multipotent neural progenitors towards replacement of missing granules neurons in meander tail cerebellum may help determine the locus of mutant gene action. Development 1997; 124: 4213-4224.

58 Yandava B, Billinghurst L, Snyder E. 'Global' cell replacement is feasible via neural stem cell transplantation: evidence from the demyelinated shiverer mouse brain. Proc Natl Acad Sci USA 1999; 96: 7029-7034.

59 Pearse DD et al. cAMP and Schwann cells promote axonal growth and function recovery after spinal cord injury. Nat Med 2004; 10: 610-616.

60 Saporta S et al. Human umbilical cord blood stem cells infusion in spinal cord injury: engraftment and beneficial influence on behavior. J Hematother Stem Cell Res 2003; 12: $271-278$.
61 Li Y, Field PM, Raisman G. Regeneration of adult rat corticospinal axons induced by transplanted olfactory ensheathing cells. J Neurosci 1998; 18: 10514-10524.

62 Moreno-Flores T, Diaz-Nido J, Wandosell F, Avila J. Olfactory ensheathing glia: drivers of axonal regeneration in the central nervous system? J Biomed Biotechnol 2002; 2: $37-43$.

$63 \mathrm{Liu} \mathrm{S}$ et al. Embryonic stem cells differentiate into oligodendrocytes and myelinate in culture after spinal cord transplantation. Proc Natl Acad Sci USA 2000; 97: 6126-6131.

64 Plant GW, Christensen CL, Oudega M, Bunge MB. Delayed transplantation of olfactory ensheathing glia promotes sparing/regeneration of supraspinal axons in the contused adult rat spinal cord. $J$ Neurotrauma 2003; 20: $1-16$.

65 Teng YD et al. Functional recovery following spinal cord injury mediated by a unique polymer scaffold seeded with neural stem cells. Proc Natl Acad Sci USA 2002; 99: 3024-3029.

66 Freed CR et al. Transplantation of embryonic dopamine neurons for severe Parkinson's disease. N Engl J Med 2001; 344: 710-719.

67 Plant G. Personal communication, 2004.

68 Guttman L. Spinal Cord Injuries. Comprehensive Management and Research, 2nd edn. Blackwell Scientific Publications: London, 1976.

69 Dimitrijevic MR, Faganel J, Sherwood AM. Spinal cord stimulation as a tool for physiological research. Appl Neurophysiol 1983; 46: 245-253.

70 Herman R, He J, D’Luzansky S, Willis W, Dilli S. Spinal cord stimulation facilitates functional walking in a chronic, incomplete spinal cord injured. Spinal Cord 2002; 40: 65-68.

71 Rosenfeld JV, Gillett GR. Ethics, stem cells and spinal cord repair. MJA 2004; 180: 637-639.

72 Bregman BS et al. Neurotrophic factors increase axonal growth after spinal cord injury and tranplantation in the adult rat. Exp Neurol 1997; 148: 475-494.

73 Jin Y, Fischer I, Tessler A, Houle JD. Transplants of fibroblasts genetically modified to express BDNF promote axonal regeneration from supraspinal neurons following chronic spinal cord injury. Exp Neurol 2002; 177: 265-275.

74 Kaelan C. An investigation of possible Tran synaptic neuronal degeneration and central nerve fiber preservation in human spinal cord injury. Master of Science thesis, University of Western Australia, 1987.

75 Clark G, Tong YC, Patrick JF. Cochlear Prostheses. Churchill Livingstone: Edinburgh, 1990.

76 Bach-y-Rita P. Theoretical aspects of sensory substitution and of neurotransmission-related reorganization in spinal cord injury. Spinal Cord 1999; 37: 465-474.

77 Schmitt AB et al. Retrograde reactions of Clarke's nucleus neurons after human spinal cord injury. Ann Neurol 2003; 54: 534-539.

78 Nacimiento W, Brook GA, Noth J. Lesion-induced neuronal reorganization in the spinal cord: morphological aspects. Brain Plasticity, Adv Neurol 1997; 73: 37-59.

79 Brook GA et al. Spontaneous longitudinally orientated axonal regeneration is associated with the Schwann cell framework within the lesion site following spinal cord compression injury of the rat. Journal of Neurosciences Research 1998; 53: 51-65. 